\title{
IVD.0I - Lessons learnt from the Plasmodium vivax MSPI towards malaria control and elimination efforts
}

Paulo Afonso Nogueira ${ }^{1 *}$; Elizangela Farias ${ }^{2}$.

1FIOCRUZ-ILMD;

2UFAM.

Introduction: Due to unique biological features, the Plasmodium vivax enhanced its ability to survive and propagate further transmission. The efforts toward malaria control to eradication of the disease should pass through identification of vivax relapses and asymptomatic malaria infections.

Objective: Amongst lessons learnt from studies with the Plasmodium vivax MSP1, we could design two novel approaches to be used as diagnostic tools that aim at reducing transmission.

Methodology: The quantification and genotyping of parasite based on MSP1 haplotypes using the new generation sequencing and comparison with a haplotype-specific serology against a broad set of Block 2 PvMSP1 peptides. The humoral response based on isotype, subclass and avidity profiles against a set of recombinant proteins PvMSP1, such as MSP1-19, N-terminal PvMSP1 and set of Block 2 PvMSP1 variants.

Results: In case of vivax relapse, we developed a methodology to quantify haplotype MSP 1 by new generation sequencing and comparing with a haplotype-specific serology against a broad set of peptides to estimate if the haplotype might be originated from hypnozoites. This concept proof needed to be compared with gold-standard microsatellite protocols during a cohort of six months to be used as molecular diagnostic tool especially to evaluate hipnozoiticidal therapies. Moreover, the potential of serologic profiles (isotype, subclass and avidity) against different domains of MSP1 to detect asymptomatic malaria infections.

Conclusion: Our lessons learnt from the PvMSP1 ensure us to provide an adequate insight into the epidemiology of malaria to aid elimination efforts.

Keywords: Plasmodium vivax; MSP1; new insight for eradication 